\title{
Drug-induced Hypersensitivity Syndrome Accompanied by Pulmonary Lesions Exhibiting Centrilobular Nodular Shadows
}

\author{
Tetsuro Sawata ${ }^{1}$, Masashi Bando ${ }^{1}$, Haruna Kogawara ${ }^{1}$, Masayuki Nakayama ${ }^{1}$, Naoko Mato ${ }^{1}$, \\ Hideaki Yamasawa ${ }^{1}$, Tamiko Takemura ${ }^{2}$ and Yukihiko Sugiyama ${ }^{1}$
}

\begin{abstract}
A 51-year-old woman diagnosed with Crohn's disease developed drug-induced hypersensitivity syndrome (DIHS) 12 and six weeks after starting the oral intake of mesalazine and trimethoprim/sulfamethoxazole, respectively. Chest CT showed centrilobular nodular shadows and a transbronchial lung biopsy (TBLB) revealed infiltration of inflammatory cells predominantly in the small pulmonary artery walls and bronchiolar walls. Regarding pulmonary lesions of DIHS, infiltrative shadows have sometimes been reported, whereas nodular shadows have rarely been documented. This is a valuable case report for considering the mechanism underlying the development of pulmonary lesions in case of DIHS.
\end{abstract}

Key words: drug-induced hypersensitivity syndrome, Crohn's disease, centrilobular nodular shadows

(Intern Med 55: 1159-1163, 2016)

(DOI: 10.2169/internalmedicine.55.5694)

\section{Introduction}

Patients with drug-induced hypersensitivity syndrome (DIHS) exhibit severe drug-induced rashes accompanied by fever and organ dysfunction 2-6 weeks after the oral intake of certain kinds of medications (1-4). It is thought that the complication rate of lung injury in DIHS patients is approximately 5\% (5). In most previous report, chest computed tomography (CT) demonstrated infiltrative shadows (1-4), and only one reported case involved centrilobular nodular shadows (6). Importantly, no reports have investigated the pulmonary lesions of patients with DIHS pathologically. We herein report the case of a patient with DIHS accompanied by pulmonary lesions who exhibited centrilobular nodular shadows on CT and was pathologically examined using a transbronchial lung biopsy (TBLB).

\section{Case Report}

A 51-year-old woman with no smoking or allergy history was diagnosed with Crohn's disease and started the oral intake of mesalazine at 3,000 mg/day and prednisolone at 40 $\mathrm{mg} /$ day immediately after diagnosis. Her gastrointestinal symptoms improved, and the dose of prednisolone was reduced to $10 \mathrm{mg} /$ day on day 21 . On day 30 , the oral intake of sulfamethoxazole/trimethoprim (ST compound) at 1tablet/ day was started. Mesalazine and prednisolone were continued. On day 72, she developed an erythematous papule and stopped taking all oral medicines the same day based on her own judgment. However, she developed a body temperature of $38.3^{\circ} \mathrm{C}$ and rash as well as general fatigue and dyspnea on exertion and, on day 80, eight days after the development of the rash, was referred to our hospital.

A physical examination revealed a height of $157.2 \mathrm{~cm}$, body weight of $36.8 \mathrm{~kg}$, blood pressure of $107 / 83 \mathrm{mmHg}$, pulse rate of 126 beats $/ \mathrm{min}$, body temperature of $36.5^{\circ} \mathrm{C}$, respiratory rate of $18 / \mathrm{min}$ and percutaneous oxygen saturation $\left(\mathrm{SpO}_{2}\right)$ of $95 \%\left(\mathrm{O}_{2} 1 \mathrm{~L} / \mathrm{min}\right.$, nasal administration). No superficial lymph nodes were palpable, whereas fine crackles were heard on inhalation in the bilateral lower lungs.

Fused erythematous papules were recognized over a wide range of the patient's face and a similar rash was observed on her chest and abdomen. Disseminating erythematous papules were noted on the dorsal surface of the hands to forearms bilaterally and spotty purpura was detected on the

${ }^{1}$ Division of Pulmonary Medicine, Department of Medicine, Jichi Medical University, Japan and ${ }^{2}$ Department of Pathology, Japanese Red Cross Medical Center, Japan

Received for publication April 30, 2015; Accepted for publication July 20, 2015

Correspondence to Dr. Tetsuro Sawata, sawata@jichi.ac.jp 
lower extremities. No enanthema or blister formation was observed (Fig. 1).

Blood tests on admission showed elevation of the white blood cell count and eosinophil fraction with the presence of atypical lymphocytes (Table). Liver and biliary enzymes were elevated, while low IgG and high IgE levels were observed. Chest CT showed faint small nodular shadows in a diffuse centrilobular pattern in the bilateral lungs (Fig. 2). After admission, bronchoalveolar lavage fluid (BALF) was collected from the right $\mathrm{B}^{5} \mathrm{~b}$. The collection rate was $52 \%$

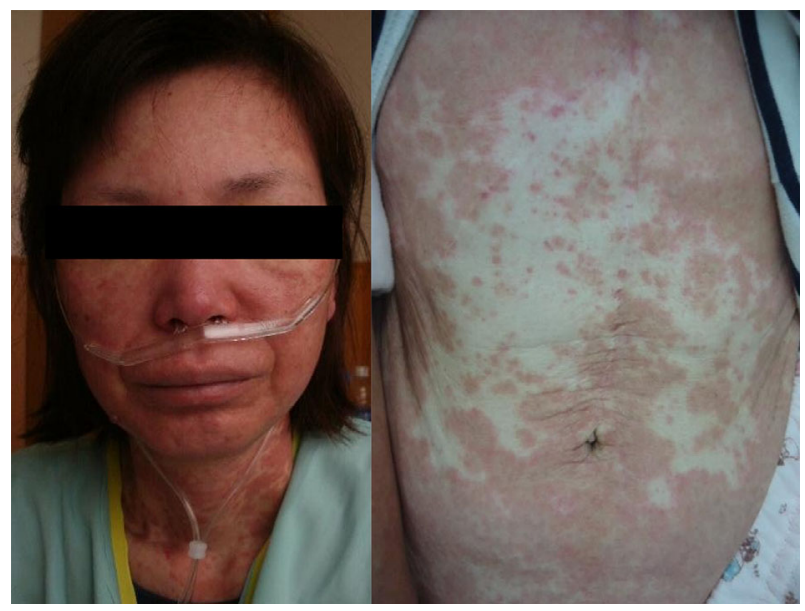

Figure 1. Fused erythematous papules were recognized in a wide range on the patient's face, and a similar rash was observed on her chest and abdomen. and the total cell count was increased at $15.2 \times 10^{5} / \mathrm{mL}$. Both lymphocyte and eosinophil fractions were increased, accounting for $36.2 \%$ and $5.5 \%$, respectively.

TBLB was performed in the right upper lobe. A histological examination revealed infiltration of inflammatory cells, consisting predominantly of lymphocytes and eosinophils, around the small pulmonary arteries and in the respiratory bronchiolar mucosa (Fig. 3). Fibrinous exudate and exfoliation of alveolar epithelial cells were observed in the alveolar lumina, in addition to the infiltration of lymphocytes and eosinophils in the alveolar walls (Fig. 4). No granulomas were observed in the lung specimens.

The titer of serum human herpesvirus 6 (HHV-6) DNA was measured in the blood sample obtained on admission, which showed less than $2.0 \times 10^{1}$ copy $/ 10^{6}$ cells; however, it increased to $5.3 \times 10^{1}$ copy $/ 10^{6}$ cells three weeks after admission. Based on the histological findings, the dose of prednisolone was increased to $20 \mathrm{mg} /$ day, and, 20 days after the rash was observed, and the patient's clinical symptoms and pulmonary imaging findings improved (Fig. 5). Therefore, the prednisolone dose was gradually tapered. We conducted a drug-induced lymphocyte stimulation test (DLST), which was positive for mesalazine, but negative for the ST compound. We considered the pulmonary lesions to be associated with DIHS based on the patient's clinical course, and she was been followed up for five months at the outpatient clinic. No deterioration in clinical symptoms or pulmonary imaging findings has been observed. The clinical course is shown in Fig. 6.

Table. Laboratory Findings on Admission.

\begin{tabular}{|c|c|c|c|c|c|}
\hline \multicolumn{2}{|l|}{ Hematology } & \multicolumn{2}{|l|}{ Serology } & \multicolumn{2}{|l|}{ BGA(nasal 1L/min) } \\
\hline WBC & $16,800 / \mu \mathrm{L}$ & $\operatorname{ESR}(1 \mathrm{hr})$ & $82 \mathrm{~mm}$ & $\mathrm{pH}$ & 7.42 \\
\hline Neut & $45.5 \%$ & CRP & $2.43 \mathrm{mg} / \mathrm{dL}$ & $\mathrm{PaCO}_{2}$ & $34.9 \mathrm{mmHg}$ \\
\hline Lym & $15.5 \%$ & IgG & $480 \mathrm{mg} / \mathrm{dL}$ & $\mathrm{PaO}_{2}$ & $72.8 \mathrm{mmHg}$ \\
\hline Eos & $31.5 \%$ & $\operatorname{IgE}$ & $3,469 \mathrm{U} / \mathrm{mL}$ & $\mathrm{HCO}_{3}^{-}$ & $22.3 \mathrm{mmol} / \mathrm{L}$ \\
\hline Baso & $0.5 \%$ & ANA & 40 times & $\mathrm{BE}$ & $-1.4 \mathrm{mmol} / \mathrm{L}$ \\
\hline Mon & $3.0 \%$ & KL-6 & $190 \mathrm{U} / \mathrm{mL}$ & & \\
\hline Atypical Lym & $4.0 \%$ & SP-D & $89 \mathrm{ng} / \mathrm{mL}$ & Sputum examination & \\
\hline $\mathrm{RBC}$ & $444 \times 10^{4} / \mu \mathrm{L}$ & Mycoplasma antibody & $<40$ times & Bacterial & $(-)$ \\
\hline $\mathrm{Hb}$ & $12.7 \mathrm{~g} / \mathrm{dL}$ & $\mathrm{T}$ spot $\mathrm{TB}$ & $(-)$ & Acid-fast bacilli & \\
\hline $\mathrm{Ht}$ & $38.8 \%$ & & & Smear & $(-)$ \\
\hline \multirow[t]{2}{*}{ Plt } & $30.1 \times 10^{4} / \mu \mathrm{L}$ & & & Culture & $(-)$ \\
\hline & & & & PCR TB & $(-)$ \\
\hline \multirow[t]{2}{*}{ Biochemistry } & & & & PCR MAC & $(-)$ \\
\hline & & & & Cytology & Class I \\
\hline $\mathrm{TP}$ & $5.2 \mathrm{~g} / \mathrm{dL}$ & & & & \\
\hline Alb & $2.6 \mathrm{~g} / \mathrm{dL}$ & & & Bronchoalveolar lavage fluid(rt $\left.\mathrm{B}^{5} \mathrm{~b}\right)$ & \\
\hline T-Bil & $0.48 \mathrm{mg} / \mathrm{dL}$ & & & Collection rate & $52 \%$ \\
\hline AST & $42 \mathrm{IU} / \mathrm{L}$ & & & Total cells & $15.2 \times 10^{5}$ \\
\hline ALT & $79 \mathrm{IU} / \mathrm{L}$ & & & Percent of total cells $(\%)$ & \\
\hline $\mathrm{LDH}$ & $419 \mathrm{IU} / \mathrm{L}$ & & & Mono & 55.5 \\
\hline ALP & $1,147 \mathrm{IU} / \mathrm{L}$ & & & Lym & 36.2 \\
\hline BUN & $15 \mathrm{mg} / \mathrm{dL}$ & & & Neut & 2.8 \\
\hline $\mathrm{Cr}$ & $0.63 \mathrm{mg} / \mathrm{dL}$ & & & Eos & 5.5 \\
\hline $\mathrm{Na}$ & $141 \mathrm{mEq} / \mathrm{L}$ & & & $\mathrm{CD} 4 / \mathrm{CD} 8$ rate & 0.43 \\
\hline $\mathrm{K}$ & $4.3 \mathrm{mEq} / \mathrm{L}$ & & & & \\
\hline \multirow[t]{3}{*}{$\mathrm{Cl}$} & $92 \mathrm{mEq} / \mathrm{L}$ & & & Drug-induced lymphocyte stimulation test & S.I $(\%)$ \\
\hline & & & & Mesalazine & 235 \\
\hline & & & & Sulfamethoxazole/trimethoprim & 81 \\
\hline
\end{tabular}




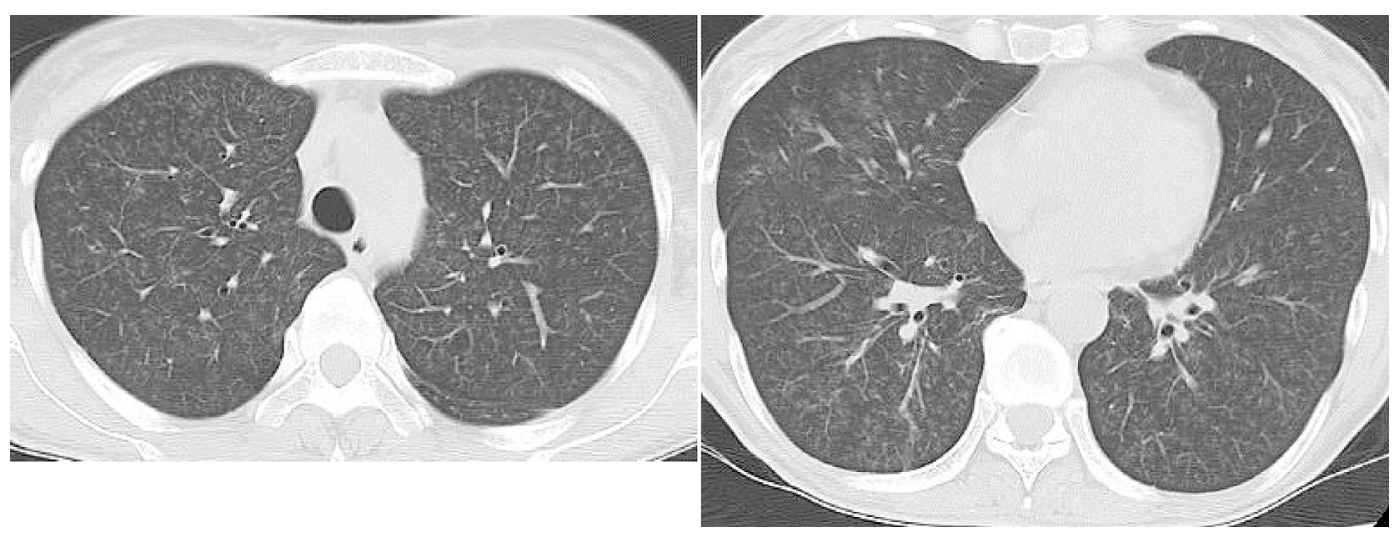

Figure 2. Faint small nodular shadows were observed diffusely in the bilateral upper, middle (lingular segment), and lower lobes of the lungs on chest CT. A centrilobular distribution pattern was recognized.

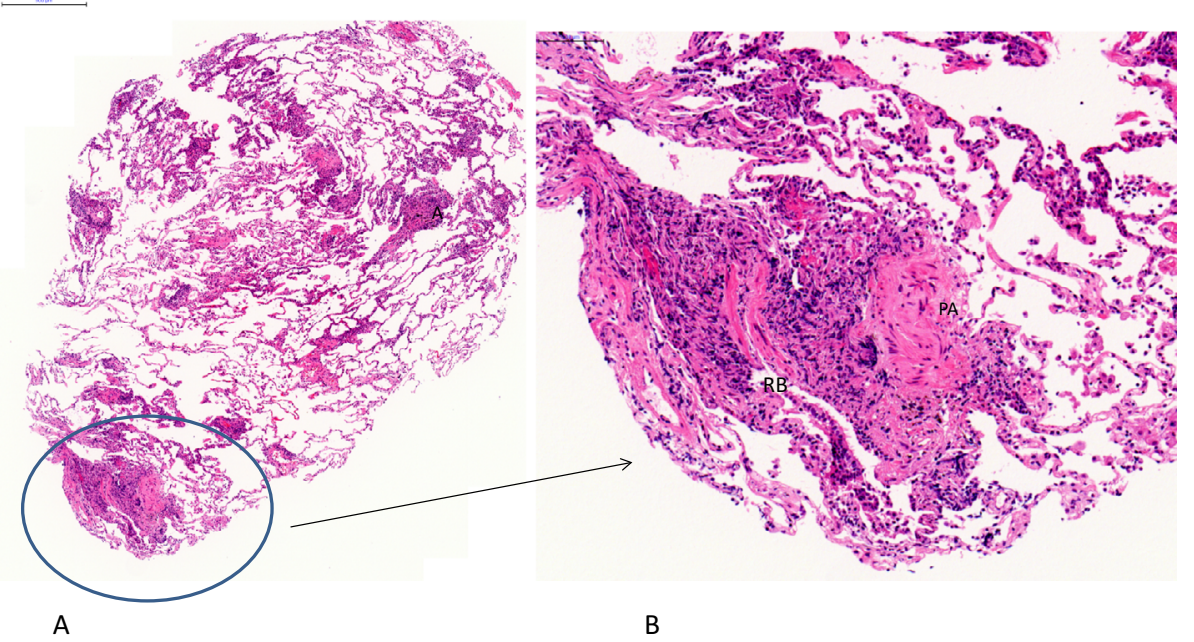

Figure 3. (A) A panoramic view of a TBLB specimen showing periarteriolar, alveolar septal and peribronchiolar inflammatory cell infiltration [Hematoxylin and Eosin $(H \& E)$ staining, $\times 5$ ]. (B) Infiltration of lymphocytes, neutrophils and eosinophils around a respiratory bronchiole in the encircled area in A (H\&E staining, ×20). RB: respiratory bronchiole, PA: pulmonary artery

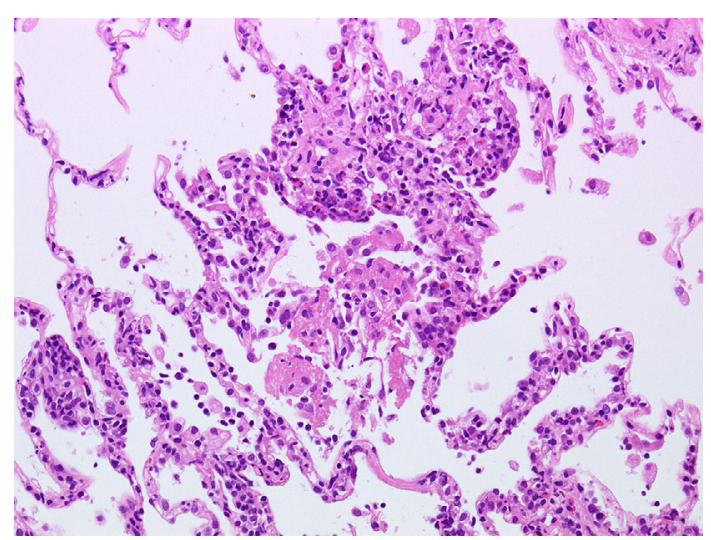

Figure 4. Exfoliation of pneumocytes, fibrinous exudate and the infiltration of lymphocytes and eosinophils in the alveolar walls in a TBLB specimen (Hematoxylin and Eosin staining, $\times 20$ ).

Discussion

The present patient met the diagnostic criteria for DIHS in Japan $(7,8)$. In the present case, CT showed centrilobular nodular shadows, and it was possible to compare the imaging findings with the pathological findings according to TBLB. It is thought that the complication rate of lung injury in DIHS patients is approximately 5\% (5), and infiltrative shadows are the most frequently observed pattern (9-12). Centrilobular nodular shadows have been reported in only one case (6). Moreover, no reports have pathologically investigated lung injury developed as a complication of DIHS in detail.

Centrilobular lesions are observed in the terminal and respiratory bronchioles and surrounding pulmonary parenchyma. The high-resolution CT (HRCT) findings of centrilobular lesions are categorized into two patterns: faint and 


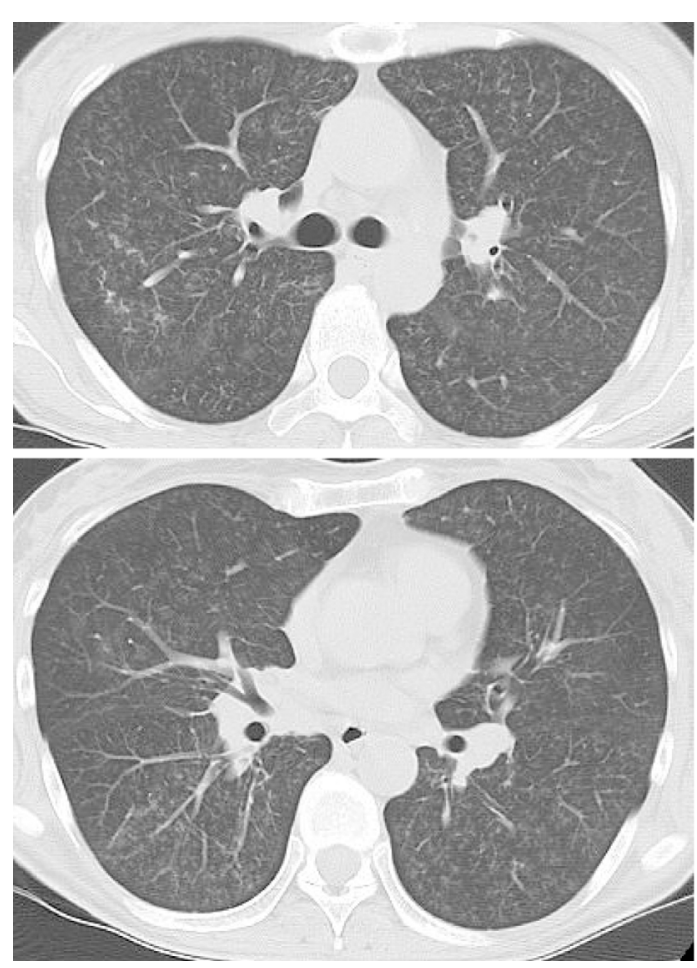

Before treatment

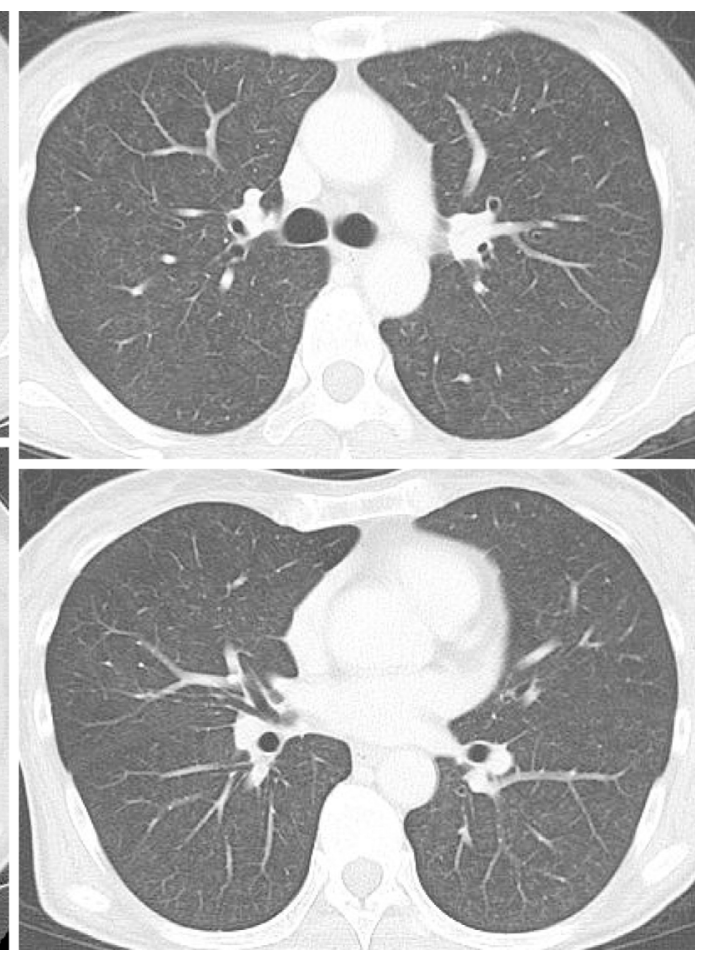

2 weeks after treatment

Figure 5. A substantial improvement was observed in these findings two weeks after initiating the treatment.

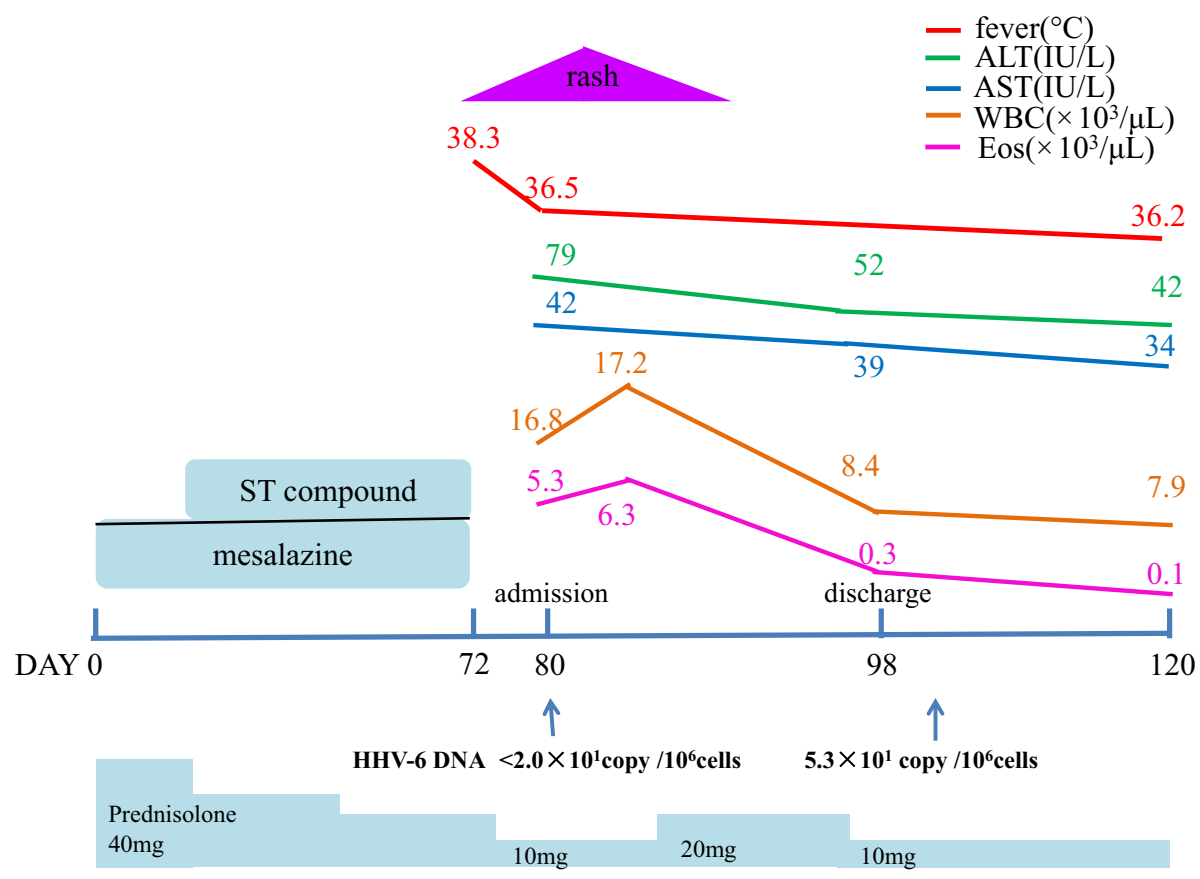

Figure 6. The patient's clinical course.

ill-delineated nodular shadows and branching shadows exhibiting a relatively high-density tree-in-bud pattern (13). The present case was categorized into the former pattern and hypersensitivity pneumonia, pneumoconiosis and transairway lesions such as alveolar hemorrhage, were considered as possible diagnoses.

It was difficult to suspect that the pulmonary lesions were a complication of DIHS based on the CT images in the pre- sent case, whereas the pathological findings of the TBLB specimens suggested an allergic reaction of the body to a medicine absorbed into the blood, which induced the spread of inflammatory cells from the pulmonary artery to the respiratory bronchiolar mucosa. These findings were evaluated as being centrilobular nodular shadows on CT. There are several reports of inflammatory bowel disease involving airway disease (14-16). In the present case, radiological and 
histological studies did not reveal any ectasis or wall thickening of the bronchioles or granuloma formation, which are commonly observed in patients with Crohn's disease. Therefore, it was unlikely that this pulmonary lesion was associated with Crohn's disease.

Drugs associated with DIHS are mostly limited to anticonvulsants, antidepressants, sulfonamides/sulfones, antiinflammatories, anti-infectives, angiotensin-converting enzyme inhibitors and beta-blockers. It has been suggested that this phenomenon is attributable to abnormal metabolic enzymes, although the precise mechanism remains unclear at present. DLST tests are considered useful for exploring medications that cause DIHS, although the timing at which the DLST is performed is critical. In DIHS patients, the test rarely shows positive results in the onset period, whereas positive results are usually noted 4-6 weeks later and remain persistently positive for more than one year after termination of the intake of the medicine. This is considered to be the characteristic pattern of DIHS (17).

Taken together, the positive result on the DLST for mesalazine in the blood sample obtained at presentation could have been a false-positive finding in the present case. Mesalazine predominantly exerts its effect in the large bowel mucosa and is generally not absorbed into the bloodstream. Therefore, mesalazine is considered to be a medicine that is unlikely to cause DIHS, and it is difficult to conclude that mesalazine induced DIHS. As for the reason for the positive DLST for mesalazine, it is suggested that the ST compound might have caused DIHS and hypersensitivity to multiple medicines, and then induced a hypersensitive reaction to mesalazine.

In the present case, it is thought that a gradual decrease in the dose of prednisolone was useful for preventing relapse of pulmonary lesions associated with DIHS. It is known that eruptions, fever and organ dysfunction may relapse after termination of the causal medicine of DIHS $(1,2)$, and two causal mechanisms of drug allergies and reactivation of HHV-6 have been elucidated $(17,18)$. It is supposed that a recovery from an immunosuppressive state associated with the termination of the causal medicine induces an immune reaction against reactivated HHV-6 and aggravates clinical symptoms.

Regarding the treatment for DIHS, it is necessary to suppress any rapid recovery of the immune system, and the systemic administration of prednisolone is effective (7). The dose of prednisolone was increased to $20 \mathrm{mg}$ /day in the present case, and a gradual decrease in the dose of prednisolone after symptomatic improvement did not cause relapse of the patient's clinical symptoms.

In conclusion, centrilobular nodular shadows on HRCT were confirmed in this patient with DIHS based on a histopathological examination of TBLB specimens. Further investigation of the pathogenesis of pulmonary manifestations of
DIHS is therefore needed.

The authors state that they have no Conflict of Interest (COI).

\section{References}

1. Suzuki $Y$, Inagi $R$, Aono $T$, Yamanishi $K$, Shiohara T. Human herpesvirus 6 infection as a risk factor for the development of severe drug-induced hypersensitivity syndrome. Arch Dermatol 134: 1108-1112, 1998.

2. Tohyama M, Yahata Y, Yasukawa M, et al. Severe hypersensitivity syndrome due to sulfasalazine associated with reactivation of human herpesvirus 6. Arch Dermatol 134: 1113-1117, 1998.

3. Hashimoto K, Yasukawa M, Tohyama M. Human herpesvirus 6 and drug allergy. Curr Opin Allergy Clin Immunol 3: 255-260, 2003.

4. Sekine N, Motokura T, Oki T, et al. Rapid loss of insulin secretion in a patient with fulminant type 1 diabetes mellitus and carbamazepine hypersensitivity syndrome. JAMA 285: 1153-1154, 2001.

5. Cacoub P, Musette P, Descamps V, et al. The DRESS syndrome: a literature review. Am J Med 124: 588-597, 2011.

6. Kikuchi Y, Horiuchi T. Bronchiolitis obliterans associated with drug-induced hypersensitivity syndrome. Igakuno Ayumi (Journal of Clinical and Experimental Medicine) 205: 976-979, 2003 (in Japanese).

7. Shiohara T, Inaoka M, Kano Y. Drug-induced hypersensitivity syndrome (DIHS): a reaction induced by a complex interplay among herpesviruses and antiviral and antidrug immune responses. Allergol Int 55: 1-8, 2006.

8. Shiohara T, Kano Y, Takahashi R. Current concepts on the diagnosis and pathogenesis of drug-induced hypersensitivity syndrome. Jpn Med Assoc J 52: 347-352, 2009.

9. Hamamoto J, Tomita Y, Uehara Y, et al. Drug-induced hypersensitivity syndrome with pulmonary infiltration: successful treatment with steroid pulse therapy. Nihon Naika Gakkai Zasshi (Journal of Japanese Society of Internal Medicine) 96: 2779-2782, 2007 (in Japanese).

10. Lee JH, Park HK, Heo J, et al. Drug Rash with Eosinophilia and Systemic Symptoms (DRESS) syndrome induced by celecoxib and anti-tuberculosis drugs. J Korean Med Sci 23: 521-525, 2008.

11. Lee SP, Kim SH, Kim TH, et al. A case of mexiletine-induced hypersensitivity syndrome presenting as eosinophilic pneumonia. J Korean Med Sci 25: 148-151, 2010.

12. Gómez-Zorrilla $S$, Ferraz AV, Pedrós $C$, Lemus $M$, Peña $C$. Levetiracetam-induced drug reaction with eosinophilia and systemic symptoms syndrome. Ann Pharmacother 46: 7-8, 2012.

13. Okada F, Ando Y, Yoshitake S, et al. Clinical/Pathological correlations in 553 patients with primary centrilobular findings on highresolution CT scan of the thorax. Chest 132: 1939-1948, 2007.

14. Vandenplas $O$, Casel S, Delos M, et al. Granulomatous bronchiolitis associated with Crohn's disease. Am J Respir Crit Care Med 158: 1676-1679, 1998.

15. Camus P, Piard F, Ashcroft T, et al. The lung in inflammatory bowel disease. Medicine (Baltimore) 72: 151-183, 1993.

16. Casey MB, Tazelaar HD, Myers JL, et al. Noninfectious lung pathology in patients with Crohn's disease. Am J Surg Pathol 27: 213-219, 2003.

17. Kano Y, Hirahara K, Mitsuyama Y, Takahashi R, Shiohara T. Utility of the lymphocyte transformation test in the diagnosis of drug sensitivity: dependence of its timing and the type of drug eruption. Allergy 62: 1439-1444, 2007.

18. Kano Y, Hirahara K, Sakuma K, Shiohara T. Several herpesviruses can reactivate in a severe drug-induced multiorgan reaction in the same sequential order as in graft-versus-host disease. Br J Dermatol 155: 301-306, 2006.

(C) 2016 The Japanese Society of Internal Medicine http://www.naika.or.jp/imonline/index.html 